\title{
Prevalence of Dirofilaria and Hemato-biochemical Effect in Street Dogs of Kathmandu Valley and Siddharthanagar Municipality, Bhairahawa, Nepal
}

\author{
S. Rimal*,1, A. Adhikari ${ }^{1}$, K. Khadka ${ }^{1}$, B. Thapa ${ }^{2}$, R. Acharya ${ }^{1}$ \\ ${ }^{1}$ Paklihawa Campus, IAAS, TU. \\ ${ }^{2}$ Central Veterinary Referral Hospital, Tripureswor \\ *Corresponding Author: rimalshikha@gmail.com
}

\begin{abstract}
A cross sectional study was conducted to determine the prevalence of dirofilariosis in street dogs of Kathmandu valley and Siddharthanagar, Rupandehi from January 2019 to March 2019. .Blood sample from 155 dogs of Kathmandu Valley and 150 from Siddharthanagar municipality were collected and examined by Wet Smear, Modified Knott Technique and Buffy Coat methods. $P C V$ value and biochemical parameters were analyzed. Data were analyzed to determine the Prevalence of Dirofilaria and correlation of infections with age, sex and biochemical parameters. The prevalence in Kathmandu valley was 0\% (0/156) while the prevalence in Siddharthanagar was $19.33 \%$, while the prevalence in direct smear, buffy coat and modified Knott technique were found to be 16\%, 16\% and 19.33\% respectively. The prevalence were significantly $(p<0.05)$ higher in old age and higher in 3-5 years of age i.e. 51.70\%.Prevalence was higher in female $(21.70 \%)$ than male $(17.3 \%)$. AST and ALT were significantly $(p<0.05)$ increased in infected dogs. Total protein, ALP, BUN, Creatinine level were elevated in the infected dogs.
\end{abstract}

Keywords: Dirofilaria, Dog, Biochemical

\section{INTRODUCTION}

Dirofilaria is an intravascular nematode parasite of the superfamily Filarioidea that consists of mostly two species Dirofilaria immitis and Dirofilaria repens affecting canine soulsby, 2012. Dirofilariosis is prevalent in tropical, subtropical and temperate region worldwide.

Transmission occurs through blood sucking mosquitoes of Aedes, Culex and Anopheles species (Soulsby, 2012). Over 60 mosquito species have been shown to be able to Dirofilaria under experimental or natural conditions (Norma et.al., 1998). The mosquito vectors that can transmit Dirofilaria commonly present in Kathmandu valley and Rupandehi are Aedes albopictus, Culex quinquefasciatus, Aedes aegypti (Darsie et.al., 1990). Development of D. immitis to L3 larval stage in mosquitoes occurs at threshold temperature $14{ }^{\circ} \mathrm{C}$ (Genchi et.al., 2010).Pathological changes in Dirofilariaimmitis has been reported in reductions of RBC, haemoglobin and PCV along with alterations of BUN, Creatinine, ALP, AST and GGT (Bahadori et.al., 2010).

The prevalence rate of heart worm in Assam and Mizoram area of Northeastern state of India is $11.38 \%$ out of 413 sample of stray dog (Borthakur et.al., 2015). The prevalence of Dirofilaria immitis in Busan, Korea in 77 stray dogs was 6.5\% (Byein et.al., 2007). Wang et.al., reported prevalence of Dirofilaria in Henan province of central China from March 2015 to Feb 2016 with 
the overall recorded seroprevalence of $D$. immitis in dogs in $13.18 \%$. In case of Nepal, Singh et. al., (2018) has reported a case study of heartworm with chylothorax at Veterinary Teaching hospital, Bhairahawa. Pradhan et.al., (2018) reported the case of Dirofilaria in anterior chamber of eye in human in Biratnagar Eye Hospital.

Dilofilaria immitis is routinely treated in canines under the guidelines prescribed by the American Heartworm Evaluation of Growth Society using the adulticidal therapy, Melarsomine. The three-dose protocol is the treatment of choice due to its increased safety and efficacy (Humpreys, 2015). Dirofilaria is also a zoonotic parasite and humans are dead end hosts. D. repens infection in human is associated with the subcutaneous lump in the affected area i.e. face and conjunctiva of the eye while human $D$. immitis infection has been associated with pulmonary dirofilariosis and is usually asymptomatic (Reddy, 2013).

In case of Nepal, there are no research published on prevalence of Dirofilaria. Climatic factors, Vectors and geographical location supports the prevalence of Dirofilaria in Nepal. Its presence have been observed in necropsy findings in 4 street dogs in Veterinary Teaching Hospital, Paklihawa, Siddharthanagar. As the disease when prevailed is complex to treat and expensive too, similarly it is a burden for public health as it is zoonotic. No vaccination practice against Dirofilaria have been seen in Nepal. So this research was mainly conducted to know the overall prevalence.

- of Dirofilaria and hemato-biochemical effect in street dogs of Kathmandu Valley and Siddharthanagar Municipality, Bhairahawa, Nepal.

\section{MATERIALS AND METHODS}

Study Area: This study was conducted in Siddharthanagar municipality, Rupandehi and Kathmandu valley. 160 samples from Kathmandu valley and 150 samples from Siddharthanagar were collected.

Collection of Blood: About $10 \mathrm{ml}$ of blood was withdrawn in sterile blood collecting vial containing EDTA. The blood was immediately transferred to a sterile vacutainer. The tube was kept under refrigeration at-4 degree celsious until further laboratory works were performed.

Techniques Used: Three methods were used as a diagnostic method for the detection of Dirofilariaas described by Taylor et.al., (2016):

Wet Smear Method: one drop of venous blood was kept on a clean microscope slide coverslip was placed. The coverslip area was examined under low magnification $(10 \times)$ of the microscope. Undulating movements of larvae can be observed.

Haematocrit Test: Fresh whole blood was drawn into a Microhematocrit tube and centrifuged for 3 minutes at $1500 \mathrm{rpm}$. The plasma portion of the separated blood was examined under low magnification $(10 \times)$. Moving microfilariae was observed in the plasma above the buffy coat. 
Modified Knott's Test: Sample of blood was drawn into a syringe containing EDTA. $1 \mathrm{~mL}$ of the blood was mixed with $9 \mathrm{~mL}$ of a $2 \%$ formalin solution and the mixture was centrifuged the mixture at $1500 \mathrm{rpm}$ for 5 minutes. Discarding the supernatant, one drop of $0.1 \%$ methylene blue was added to the sediment and mixed well. The stained sediment was transferred to a microscope slide using a Pasteur pipette to collect the entire sample and examined using the $10 \times$ microscope objective. Microfilariae was observed in an extended position with nuclei stained blue.

Packed Cell Volume Determination: PCV was determined by microhematocrit tube to separate cells from plasma.

Biochemical Test: Serum biochemical values were determined by using Autozyme reagent of Accurex Biomedical PVT. LTD. and Advanced Microprocessor Based Colorimeter.

Data Analysis: Statistical analysis was conducted using Statistical package on social science (SPSS) v. 25 with chi square test, one sample T test and compare mean and MS excel was also used for data presentation.

\section{RESULT}

\section{Overall Prevalence of Dirofilaria:}

Overall prevalence in Kathmandu valley was found to be zero while $19.33 \%$ was found to be positive among 150 samples in Siddharthanagar.

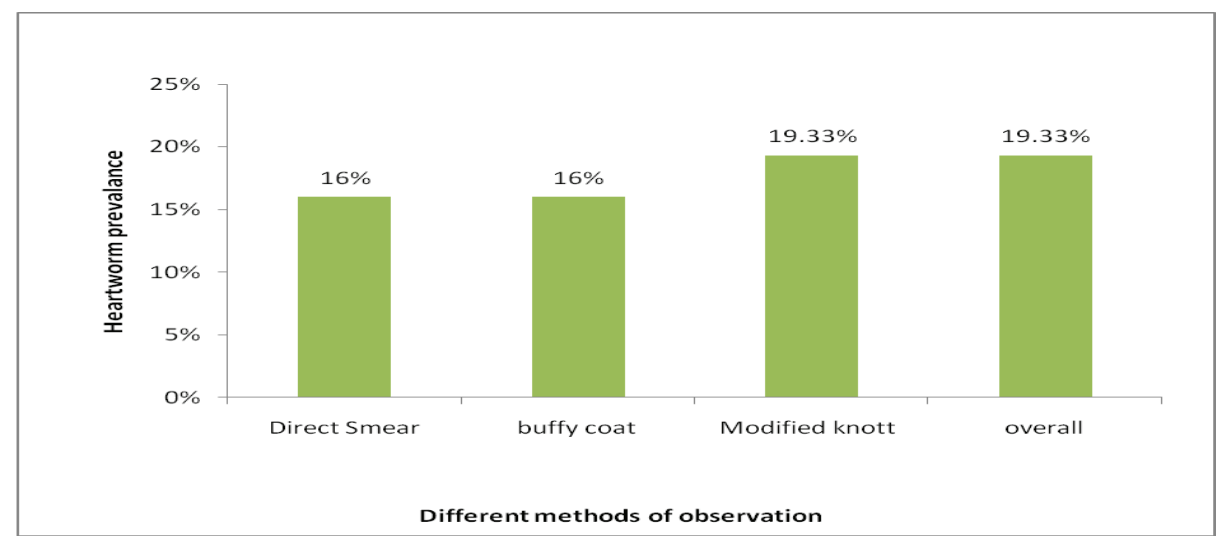

Fig. 1 Prevalence of heartworm infection in street dog of Bhairahawa in 2019

\section{Sex-wise Prevalence of Dirofilaria:}

This study revealed the prevalence in male $17.3 \%$ and in female was $21.70 \%$ among the infected dogs out of 150 samples of Siddharthanagar. 


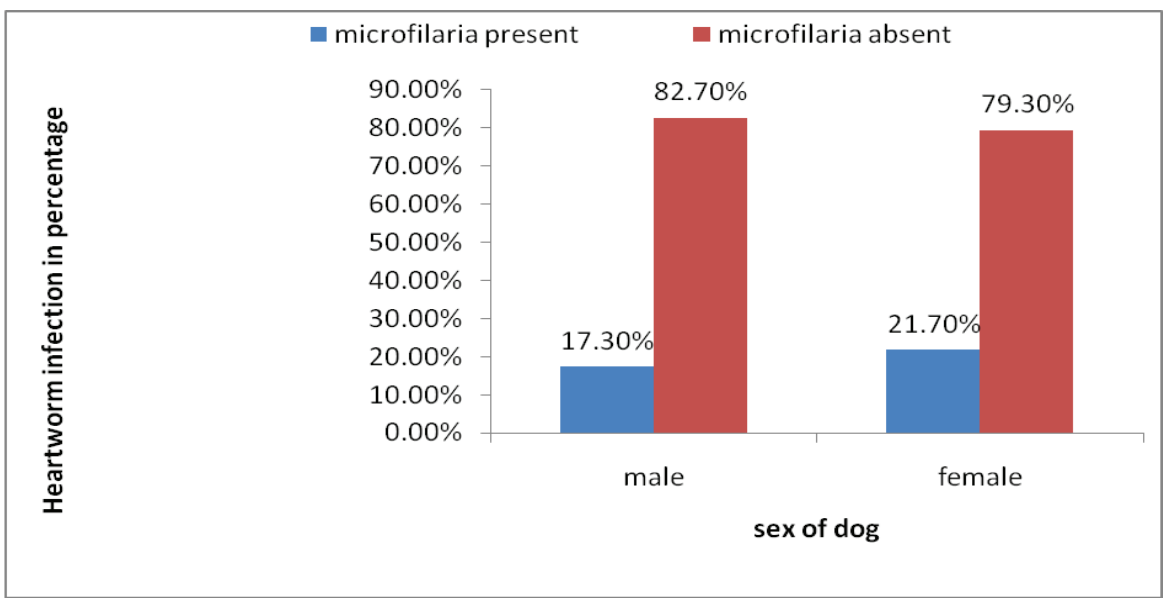

Fig. 2 Heartworm infection in male and female dog on Bhairahawa in 2019

\section{Age-wise Prevalence of Dirofilaria:}

Out of 150 samples of siddharthanagar 3.50\% were from age below 1 year, $10.30 \%$ from $1-5$ year, $51.70 \%$ between $3-5$ years and $34.50 \%$ greater than 5 year.

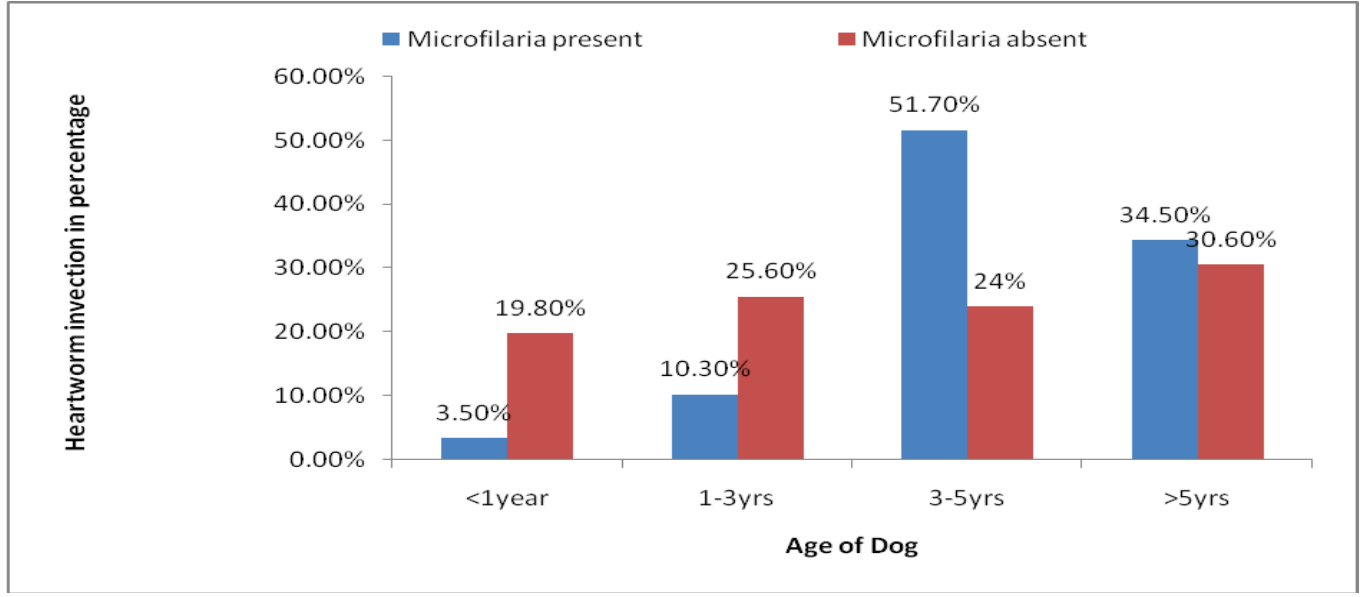

Fig. 3 Heartworm infection against different age of dog on Bhairahawa in 2019

\section{Variation of Prevalence with the Tests:}

The prevalence of heartworm microfilaria on direct smear, buffy coat method and modified Knott smear were $16 \%, 16 \%$ and $19.33 \%$ respectively on Siddharthanagar municipality, Bhairahawa.

\section{Temperature Variation:}

Of the total infected dog, $13.80 \%$ have temperature greater than 102.5 degree $\mathrm{F}$ while $86.20 \%$ have the range between 99.5-102.5 degree $\mathrm{F}$. 


\section{Haematological and Biochemical Variations:}

Chi square test shows PCV, Total protein, ALP, Urea, BUN and creatinine value insignificant with infection while AST (IU/L) and ALT (IU/L) value were found to be significant with mean value 26.73 and 27.25 in infected dogs and 17.98 and 19.91 in non infected dogs respectively. There was overall increase in AST, ALT, BUN, creatinine and Total protein level in infected dogs.

\begin{tabular}{|c|c|c|c|c|c|}
\hline S.N. & Parameter & Infected dog & Non infected dog & $P$ value & $\mathrm{T}$ value \\
\hline 1 & Total protein $(\mathrm{mg} / \mathrm{dl})$ & $7.37 \pm 0.36$ & $6.56 \pm 0.29$ & $\mathrm{p}>0.05$ & 1.166 \\
\hline 2 & $\operatorname{AST}(\mathrm{IU} / \mathrm{L})$ & $26.73 \pm 3.54$ & $17.98 \pm 2.52$ & $\mathrm{P}<0.05$ & 2.013 \\
\hline 3 & $\operatorname{ALP}(\mathrm{IU} / \mathrm{L})$ & $376.11 \pm 39.60$ & $337.35 \pm 53.71$ & $p>0.05$ & 0.581 \\
\hline 4 & ALT (IU/L) & $27.25 \pm 2.65$ & $19.91 \pm 2.47$ & $\mathrm{P}<0.05$ & 0.049 \\
\hline 5 & Urea (mg/dl) & $22.19 .12 \pm 1.91$ & $25.24 \pm 2.90$ & $\mathrm{P}>0.05$ & -0.876 \\
\hline 6 & BUN (mg/dl) & $11.39 \pm 01.01$ & $12.21 \pm 1.49$ & $\mathrm{P}>0.05$ & -0.509 \\
\hline 7 & Creatinine $(\mathrm{mg} / \mathrm{dl})$ & $1.63 \pm 0.132$ & $1.46 \pm 0.12$ & $p>0.05$ & 0.934 \\
\hline
\end{tabular}

\section{DISCUSSION}

The prevalence of Dirofilaria in our study in Siddharthanagar is lower than the findings of Retnasbapathy, 1976 i.e. 25.8\% in Malaysia and Vieira et.al.,2014 in Portugal with prevalence $27.3 \%$. However the prevalence is higher than the findings of Borthukar et.al., 2014 i.e. 11.38\% in Assam, India and Wang et.al.,2015 with prevalence of 13.18\% in Henan, Central China. This may be due to the fact that transmission of dirofilariosis is dependent upon the presence of sufficient numbers of infected, microfilaraemic dogs, susceptible mosquitoes, and a suitable climate to permit extrinsic incubation of Dirofilaria in the mosquito intermediate host (Medlock et.al., 2007, Genchi et.al., 2009).

Biochemical variationin infected dogs is similar to Zalvaris et.al., 2008 i.e Increase in AST (54.84\%), ALT (51.61\%), Creatinine (54.84\%), Total protein ( $25.81 \%)$, Urea (32.26\%) out of 31 infected dogs in Greece. Our findings also coincides with findings of Hashem et.al., 2007 in Egypt who found among 14\% infected dogs there was increase in AST, ALT, BUN, Creatinine and Total protein level. Increase in AST and ALT level may be due to loss of renal function while increase in BUN and creatinine level may be due to renal damage result of glomerulonephritis (Zalvaris et.al., 2008 , Hashem et.al., 2007).

In this study among the positive sample of Siddharthanagar, prevalence of male was $17.3 \%$ and female was $21.70 \%$. This finding is similar to the findings of Adanir et al. where prevalence rate was $22.8 \%$ in male and $21.2 \%$ in female. It was higher in the study of Yaman et.al., 2009 in Turkey where prevalence rate in male was $24.5 \%$ and female $33.3 \%$. The difference in male and female infection is not statistically significant, difference may be due to the sample size taken fro both population (Zarei et.al., 2016). 
In this study, prevalence was higher in age group 3-5 years (51.70\%).Prevalence was also in higher in old age dogs.Adanir et al findings shows higher prevalence og age greater than 7 years I.e $53.3 \%$. The fiindings of Boonyapakan where the prevalence was higher in 3-6 years of age $33.3 \%$ while in old age greater than 10 years was $41.5 \%$. The greater infection rate in older dogs (more than two years old) than younger dogs was possibly due to the longer exposure of older dogs to the mosquito bite (Zarei et.al., 2016).

Prevalence of Dirofilaria was found to be negative in Kathmandu Valley despite being favourable temperature for the parasite, presence of vector and large population of dog. However it cannot be said it is $100 \%$ negative. Further research is to be carried out taking large population size and using antigen test kit with high sensitivity and specificity.

\section{CONCLUSION}

Our study revealed the prevalence of Dirofilaria for the first time in Nepal. Prevalence of Dirofilaria in Siddharthanagar i.e. $19.33 \%$ is an alarming situation as in Nepal there is no vaccination practice against Dirofilaria. Although there was no prevalence in Kathmandu valley, a more detail study is to be carried out. Transmission of disease can take place to the major cities of Nepal as they have favourable environment, favourable temperature and presence of vectors. A holistic approach is required for the prevention in early stage and this will require the active involvement and cooperation of veterinary, allied professionals and government at all levels.

\section{REFERENCES}

DK Singh, S. Rimal, A. Adhikari, K. Singh ; Chylothorax due to Heartworm ( Dirofilariasis) in dog; The Blue cross Volume: 15 (2018).

Maharjan S., Regmi R.P. (2014). Grid Based Temperature and Relative Humidity Distribution Map of the Kathmandu Valley. Journal of Institute of Science and Technology, 19 (1): 713, [Retrieved from https://www.nepjol.info/index.php/JIST/article/view/13819/11197].

Medlock, J.M., Barras, I., Kerrod, E., Taylor, M.A., Leach, S., 2007. Analysis of climatic predictions for extrinsic incubation of Dirofilaria in theUnited Kingdom. Vector Borne Zoonotic Dis. 7, 4-14[online].[Retrieved from: https://www.ncbi.nlm.nih.gov/pubmed/17417953].

M Hashem, A Badawy. Hematological and biochemical studies on filariasis of dogs. The Internet Journal of Veterinary Medicine. 2007 Volume 4 Number 2.[Retrieved from http://print.ispub.com/api/0/ispub-article/3275]

Retnasabapathy, A and T San, K. (1976). Incidence of canine heartworm (Dirofilaria immitis) in Malaysia. The Veterinary record. 98. 68-9. [online] [Retrieved from https:/www.researchgate.net/publication/22210849]

Sanjay kumar borthakur et.al., Prevalence and Molecular Epidemiological Data on Dirofilaria immitis in Dogs from Northeastern States of India; ScientificWorldJournal. 2015; 2015: 265385 [Available:https://www.ncbi.nlm.nih.gov/pmc/articles/PMC4320797/]

Soulsby E. J. L. Helminths, Arthropods and Protozoa of Domesticated Animals. 7th. London, UK: ELBS Bailliere and Tindall; 1982.

Taylor M.A., Coop, R.L. (2016). Veterinary Parasitology. United Kingdom.Wiley-Blackwell. 
Vieira, A. L., Vieira, M. J., Oliveira, J. M., Simões, A. R., Diez-Baños, P., and Gestal, J. (2014). Prevalence of canine heartworm (Dirofilaria immitis) disease in dogs of central Portugal. Parasite (Paris, France), 21, 5. [Retrieved from https://www.ncbi.nlm.nih.gov/pmc/articles/PMC3927308]

Wang, S., Zhang, N., Zhang, Z., Wang, D., Yao, Z., Zhang, H., Liu, S. (2016). Prevalence of Dirofilaria immitis infection in dogs in Henan province, central China.. Parasite (Paris, France), 23, 43[online].[Retrieved from https://www.ncbi.nlm.nih.gov/pmc/articles/PMC5782855/].

Yaman, M andGuzel, Murat andKoltas, I.S. and Demirkazik, M andAktas, H. (2009). Prevalence of Dirofilariaimmitis in dogs from Hatay province, Turkey. Journal of helminthology. 83. 255-60. [Retrieved from https://www.researchgate.net/publication/23953145]

Zavlaris, M and E Smaragda, KoukeriandVasile, Cozma. (2019). Study on the Hematological and Biochemical Changes in Dogs INFECTED BY Dirofilaria Immitis. 